\title{
BMJ Open Improving mesothelioma follow-up care in the UK: a qualitative study to build a multidisciplinary pyramid of care approach
}

\author{
Zoe Davey (D) , Catherine Henshall
}

To cite: Davey Z, Henshall C. Improving mesothelioma follow-up care in the UK: a qualitative study to build a multidisciplinary pyramid of care approach. BMJ Open 2021;11:e048394. doi:10.1136/ bmjopen-2020-048394

- Prepublication history for this paper is available online. To view these files, please visit the journal online (http://dx.doi. org/10.1136/bmjopen-2020048394).

Received 12 January 202 Accepted 20 August 2021

\section{Check for updates}

(c) Author(s) (or their employer(s)) 2021. Re-use permitted under CC BY-NC. No commercial re-use. See rights and permissions. Published by BMJ.

Faculty of Health and Life Sciences, Oxford Brookes University, Oxford, UK

Correspondence to

Dr Zoe Davey;

zdavey@brookes.ac.uk

\section{ABSTRACT}

Objectives The findings reported in this manuscript are part of a wider study that aimed to explore mesothelioma patients' experiences of follow-up care. The aim of this phase of the study was to co-produce recommendations for policy and practice and to propose a revised, patientfocused, mesothelioma follow-up care service.

Design The consultation phase was qualitative and consisted of three group discussions with separate stakeholder groups allowing for different priorities and needs for follow-up care to be compared. An implicit approach to consensus was adopted and data were analysed iteratively using the framework method.

Setting The study was conducted in three National Health Service Trusts in the South of England. Two were secondary care settings and the third was a tertiary centre. Participants The consultation exercise comprised three group discussions with key stakeholders $(n=35)$ : mesothelioma specialist nurses $(n=9)$, mesothelioma patients and carers $(n=11)$ and local clinical commissioning group members $(n=15)$.

Results Recommendations for mesothelioma follow-up care were developed using a co-production approach and highlighted the importance of continuity of care, the provision of timely information and the central role played by mesothelioma specialist nurses, supported by the wider multidisciplinary team. Recommendations were produced together with two bespoke infographics to maximise impact and facilitate patient and public engagement with the study.

Conclusions The recommendations developed are the first that specifically examine best practice for the follow-up care pathway for mesothelioma patients. Coproduction and public engagement are crucial to priority setting develop and optimising patient-centred care.

Combining the recommendations produced with a targeted dissemination strategy and well-designed, patient-focused infographics will maximise opportunities for impact at a regional and national level.

\section{INTRODUCTION}

Malignant pleural mesothelioma (MPM) is a rare and incurable cancer mainly arising from previous occupational or environmental asbestos exposure. ${ }^{1}$ Affecting the pleural lining of the lung, MPM has a 20-50-year

\section{Strengths and limitations of this study}

To the best of our knowledge, the recommendations developed as part of the current study are the first that specifically examine best practice for the followup care pathway for patients with mesothelioma.

- Using a co-production approach allowed for the needs and priorities of key stakeholders, including patients and healthcare professionals, to be embedded into the recommendations, ensuring their feasibility and acceptability.

- Using a targeted dissemination strategy and welldesigned infographics to complement and enhance the recommendations developed will maximise their impact regionally and nationally.

- The co-production, priority setting and consensus development processes adopted were not formally evaluated in this study. Future research should examine the extent to which the recommendations produced are implemented and the degree to which this improves patient experiences of follow-up care.

latency period and as such will remain a global health concern for years despite more recent regulation of asbestos use worldwide. ${ }^{2}$ Although relatively small compared with lung cancer ( 47000 diagnoses per year) ${ }^{3}$ the UK has the highest incidence of MPM in the world (4.4/100 000 in 2017; amounting to $\sim 2700$ diagnoses per year). ${ }^{4}$ The prognosis of MPM is poor with a median survival of 8-14 months from diagnosis. ${ }^{5}$ Treatment options are limited and the symptom burden is high, most often characterised by progressive dyspnoea, chest wall pain, weight loss, sweating and fatigue. ${ }^{6-9}$ This can lead to high levels of psychological distress for the patient, with symptoms of depression, anxiety, fear and anger, all being reported. ${ }^{6} 810-15$ Moreover, unmet psychosocial and informational needs are common in MPM, and distinct psychosocial care needs, especially around feelings of hopelessness and uncertainty, 
legal and financial issues, and blame, in comparison to advanced lung cancer have been identified. ${ }^{2} 10$

Despite their unique and often complex care needs, patients with MPM regularly enter the same follow-up pathways as people with lung cancer. However, there is increasing recognition that separate assessment and care pathways should be developed to address the specific care needs of patients with MPM, and that specialist services, such as mesothelioma multidisciplinary teams, access to early supportive care and improved communication and information can provide real benefit. ${ }^{121016}$ While specialist mesothelioma nurses have been introduced in the UK, this workforce is small and thus is constrained in its ability to reach all patients with MPM needing support. ${ }^{17}$ Moreover, follow-up pathways for patients with MPM are not consistent with regards to treatment and follow-up services offered across UK National Health Service (NHS) Trusts.

The 2018 British Thoracic Society guidelines advise that patients with MPM should be provided with accurate and understandable information and have time to discuss their disease and any concerns with their healthcare professionals. In addition, 3-4 monthly follow-ups with an oncologist, respiratory physician or specialist nurse in line with individual patient treatment plans is recommended. ${ }^{2}{ }^{18}$ Despite this guidance, no national recommendations exist that specifically examine the follow-up care pathway, or focus on the supportive care needs and priorities of patients with MPM.

Adopting a collaborative approach through co-production in healthcare, whereby patient and other stakeholder expertise and experience is recognised, can empower patients, increase confidence, facilitate communication and improve patient care. ${ }^{19} 20$ A recent review on patient engagement in priority setting for healthcare services highlighted the importance of patient and public involvement in planning and designing services, informing clinical decision-making and determining health research priorities. ${ }^{21}$ Both deliberative and collaborative public and patient engagement priority setting processes involving gathering, analysing and prioritising key topics through discussion with key stakeholders, including patients, researchers and clinicians, have demonstrated positive outcomes across a range of healthcare areas. ${ }^{212}$

This paper reports on the development of co-produced recommendations for the follow-up care of patients with MPM. The recommendations were produced as part of a wider qualitative study that aimed to explore mesothelioma patients' experiences of follow-up care in three NHS Trusts in the South of England. ${ }^{23}$ This wider study adopted a qualitative design and comprised three interlinked phases: documentary analysis, interviews with patients with MPM and consultation group discussions with stakeholders. Specific objectives were to gain a detailed understanding of current mesothelioma follow-up care pathways and processes, compare findings across different trusts and to develop recommendations to propose a revised, patient-focused, follow-up care service.
Findings from the first two phases of the study (documentary analysis and interviews) have been described elsewhere in detail. ${ }^{23}$ In summary, five key themes relating to patient follow-up care were identified: people, processes, places, purpose and perception of care. There were clear variations in the access patients had to different information, resources, services, support groups and research or clinical trial, as well as in the quality and consistency of communication and care received by patients with MPM from nurses and the wider multidisciplinary team.

Here, we present the findings of the third consultation phase of this wider study, and the development of key recommendations arising from the first two phases. The aim of the final consultation phase of the study was to co-produce recommendations for policy and practice and to propose a revised, patient-focused, mesothelioma follow-up care service.

\section{METHODS}

\section{Study design}

The findings reported here are from the final consultation phase of a wider study. Both the consultation phase and the wider study were qualitative in design. The consultation phase comprised consultation group discussions with key stakeholders.

\section{Setting}

The wider study explored experiences of mesothelioma follow-up care in three NHS Trusts in the South of England. The consultation phase was carried out regionally with key stakeholders from the same network of NHS Trusts in the South of England as well as stakeholders from other regions within the UK, allowing for the provision of follow-up care to be explored at a national level.

\section{Participants}

Three key stakeholder groups were identified for the consultation exercise: mesothelioma specialist nurses; patients with MPM and carers; and local clinical commissioning group members, comprising both clinical (respiratory and oncology consultants and clinical nurse specialists) and non-clinical staff. A member of the research team attended regional and national meetings to invite stakeholder representatives from the mesothelioma specialist nurse and local clinical commissioning groups to the consultation phase. Patients who had participated in the wider study and their carers/family members were invited to take part in the consultation phase following the completion of the phase two interviews.

\section{Inclusion/exclusion}

Patient participants were eligible to take part in the wider study if they were aged 18 years and over, had been diagnosed with MPM at one of the participating trusts and were in follow-up. Patients who were non-English speaking, unable to provide written informed consent or whom the recruiting nurse specialists felt might find 
participation too distressing were excluded. Further details about the recruitment of participants to the wider study are reported elsewhere.

\section{Data collection}

Stakeholders were presented with a summary of the interview findings and were then encouraged to take part in a group discussion examining any needs, barriers and potential solutions to these issues in relation to their perceived impact of the findings on overall patient care. Topic guides addressing the themes emerging from the earlier stages of the study were used to guide the discussions between stakeholders and researchers in each of the consultation group discussions, and included questions such as 'how do these issues relate to you experience (clinical/of follow-up care)?', 'what tools/strategies/practical solutions could be useful to address these?' and 'what are the key considerations/ which issues are most important?'. Priority setting activities were undertaken to examine the relevance and importance of the themes developed from the wider study to patients.

Meetings were carried out in the following order to allow the findings of each to inform the discussions in the next, and for the recommendations to be developed iteratively: mesothelioma specialist nurses; patients and carers; and local clinical commissioning group members. Each group discussion lasted 1-3 hours and was held in a location convenient to the stakeholder group. A member of the research team took written notes throughout the meetings. In addition, each consultation group discussion was audio recorded and written informed consent was taken prior to the discussion commencing. Refreshments were provided, and travel expenses were reimbursed.

All consultation group discussions were held 1-3 months after the phase two interviews were completed.

\section{Data analysis}

An implicit approach to consensus was adopted, with two members of the research team ( $\mathrm{ZD}$ and $\mathrm{CH}$ ) meeting regularly to discuss the issues raised at each consultation group discussion, read back on written notes and listen to audio recordings. Analysis was iterative, and was carried out after each consultation group discussion, allowing for the findings from each to inform the next. The key points arising from the discussions and activities were charted using the framework method for analysis. ${ }^{24}{ }^{25}$ This allowed for the opinions, priorities and needs of the different stakeholder groups to be compared and organised in line with the key themes identified in the wider study. The research team was then able to use these findings to translate these priorities into key aspects of mesothelioma follow-up care to develop patient-focused recommendations for mesothelioma follow-up services.

\section{Infographics}

In order to maximise the potential impact of the recommendations on mesothelioma follow-up care pathways both regionally and nationally, the dissemination strategy also included the development of two infographics. When carefully designed, taking into account the target audience, narrative, key messages and aesthetics, infographics can increase both understanding and reach of research. ${ }^{26}$ The infographics for this study were developed using an interdisciplinary approach, involving members of the research team, PPI representatives, an expert in graphic design and science communication, and Mesothelioma UK.

\section{Patient and public involvement}

Patient and carer representatives (outside of the consultation group discussions) were involved in the study process throughout and were invited members of the study's Steering Group Committee. The study was funded by Mesothelioma UK, who provided access to Mesothelioma UK nurse specialists for the first of the three consultation group discussions. Mesothelioma UK reviewed and agreed both the recommendations arising from the study and the infographics, and supported the submission of the findings for publication.

\section{RESULTS}

In total, three consultation group discussions were carried out with 35 key stakeholders: mesothelioma specialist nurses $(n=9)$, patients with MPM and carers $(n=11)$ and local clinical commissioning group members, comprising respiratory and oncology consultants, clinical nurse specialists and non-clinical staff $(n=15)$. Findings from the consultation group discussions on the needs, barriers and solutions emerging from the five key themes (people, purpose, place, process and perception of care) identified in the wider study have been presented elsewhere. ${ }^{23}$ These findings highlight the importance of a specialist respiratory led team integrated shared care, the provision of relevant, evidence-based and timely information, resources, and access to support, and clear communication and continuity of care between secondary, community and palliative care services throughout the follow-up care pathway.

The core elements of these findings were subsequently translated into six key aspects of mesothelioma follow-up care: the preferred structure of the clinical care team; the timing of information provided to patients; the content of information provided to patients; the integration of secondary, palliative and community care services; the quality of additional resources made available to patients; and links between secondary care wider support services. These aspects of care were developed into six overarching recommendations. For clarity, each recommendation is accompanied by detailed subsidiary recommendations. These are presented in table 1 . 


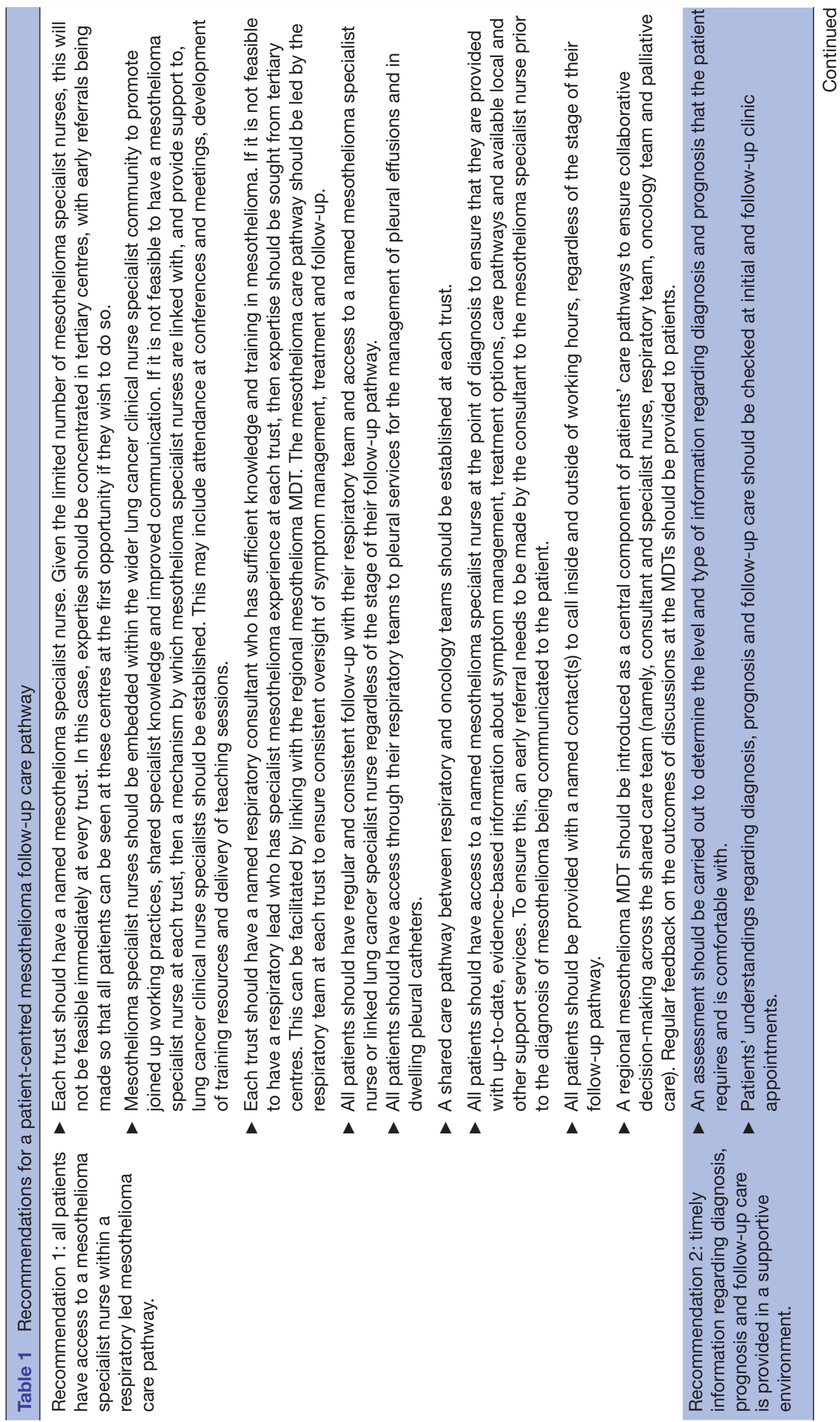




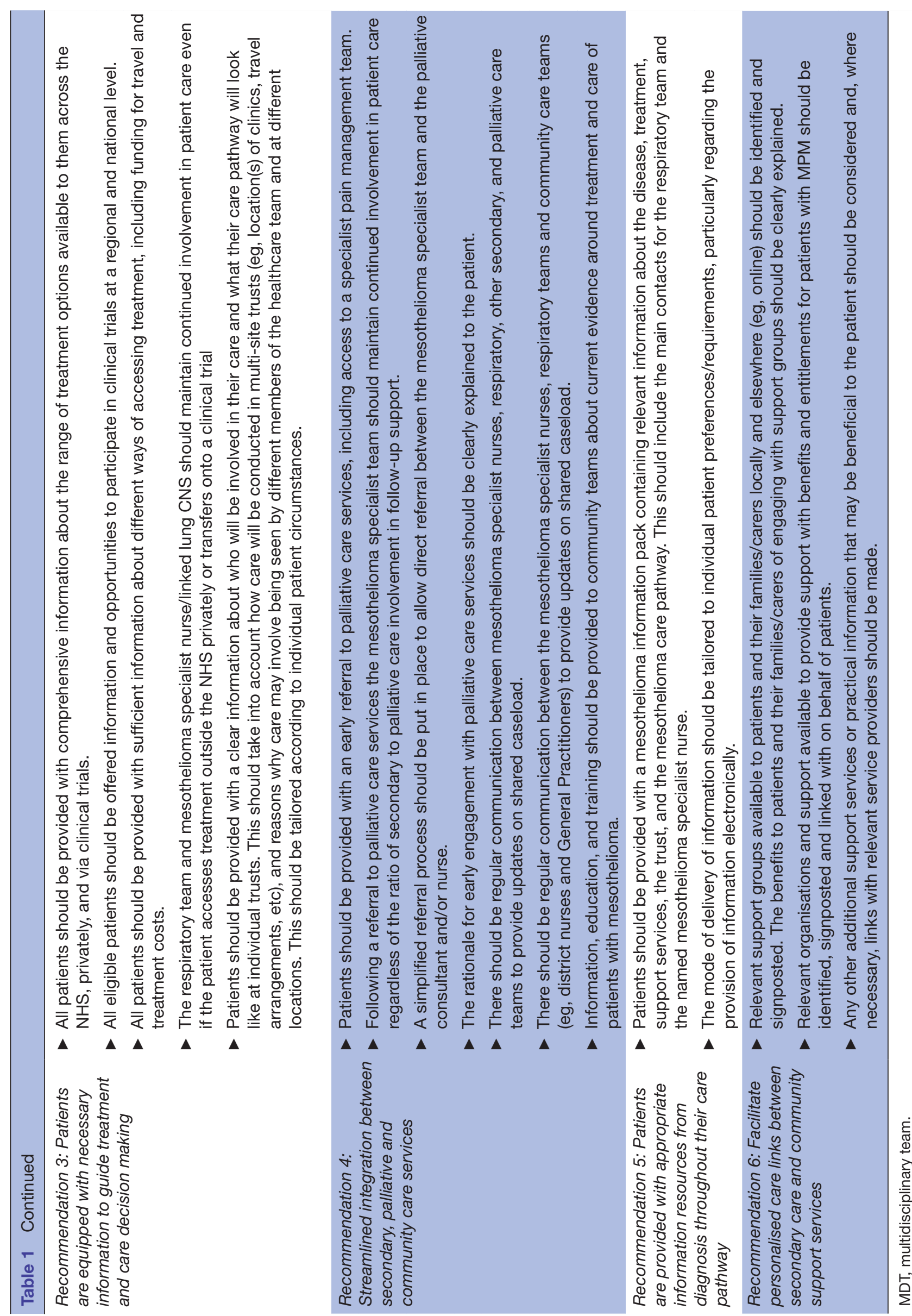


A common thread throughout the discussions with key stakeholders was the importance of continuity of care, both for the provision of high-quality care but also of instilling confidence in the care pathway. In particular, the consultation group discussion with patients and carers highlighted the importance of mesothelioma specialist nurses in providing this continuity, as well as functioning as a valuable patient advocate and resource for relevant and up-to-date information on treatment, research, accessing services and available support. As such, the core recommendation was for all patients with MPM to have access to a mesothelioma specialist nurse within a respiratory led mesothelioma care pathway (recommendation $1)$.

The importance of how and where timely, relevant information about diagnosis, prognosis and follow-up care is sensitively and appropriately communicated with patients was also particularly important to patient stakeholders (recommendation 2). The extent to which patients feel they have access to up-to-date information to guide decision-making around treatment and care was also viewed as an important priority area (recommendation 3). In particular, access to information about and routes into clinical trials and the full range of treatment options available both within and outside of the NHS was indicated, and again points to the need for specialist mesothelioma knowledge within the clinical care team. It is important that communication is supported by the provision of appropriate resources and information packs from diagnosis throughout the care pathway (recommendation 5). However, it is worthwhile noting that, although both valuable, the communication of information was prioritised over the provision of information resources, highlighting the importance of continuity of care and the relationships developed between patients and their care team.

Lastly, stakeholders highlighted the importance of palliative and community care (recommendation 4) and support services (recommendation 6), but identified the need for this to be joined up with secondary care services, reiterating the importance of continuity of care at all stages of the follow-up pathway.

The development and prioritisation of the recommendations also gave rise to the development of the "pyramid of care' as a way of conceptualising the structure of a sustainable patient-centred mesothelioma follow-up care pathway and the wider structures and core mechanisms underpinning this. The pyramid of care places a named mesothelioma specialist nurse at the top of the pyramid, supported by expertise and resource from a named respiratory consultant and the wider multidisciplinary team. It underlines the importance of well-defined relationships and clear, two-way communication and feedback channels between different clinical and support services to promote and sustain continuity of care within a patientcentred pathway. A preferred mesothelioma follow-up care pathway would give patients access to each layer of the pyramid, from the essential secondary care team at

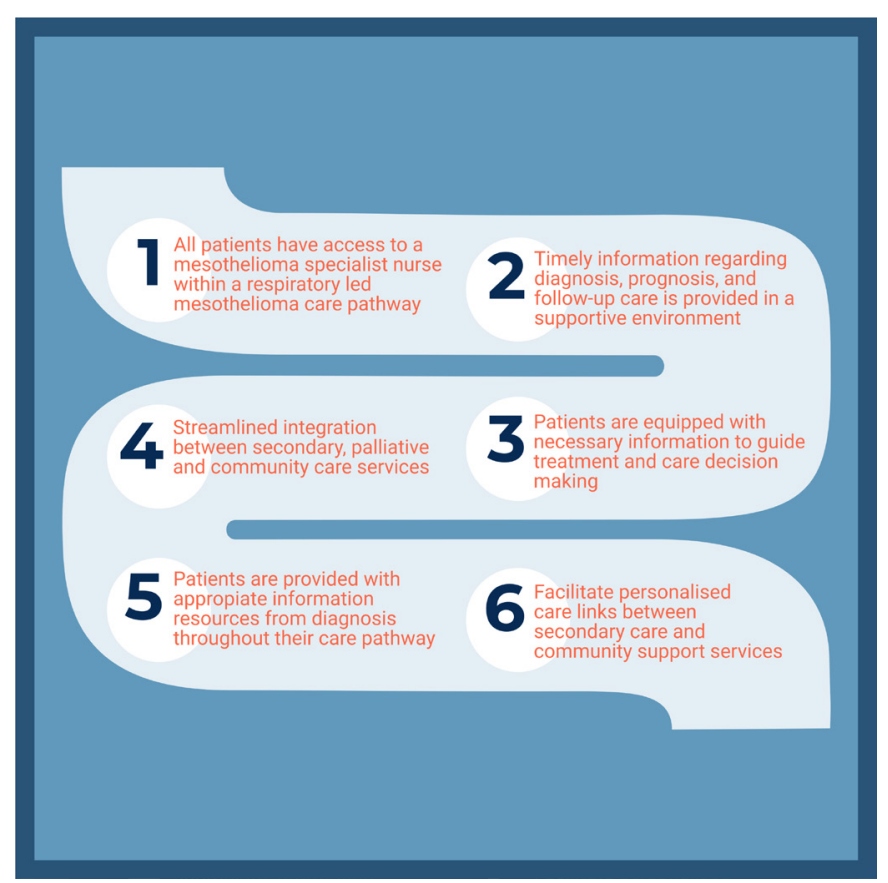

Figure 1 Recommendations for mesothelioma follow-up care infographic.

the top to the various other clinical and support services that underpin this, providing patients with an integrated care pathway throughout their entire follow-up period, including once they have been discharged into the community. Full access to the pyramid of care would engender patient satisfaction and improve patient experiences of follow-up care. In order to ensure the relevance, clarity and feasibility of the both the recommendations and the pyramid of care, feedback from Mesothelioma UK and study PPI representatives was also received on early drafts of these documents.

Two infographics were designed to represent both the pyramid of care and the key recommendations (figures 1 and 2). They were designed so as to effectively communicate the key messages from both the pyramid of care and key recommendations, enhanced by clear visuals and a strong design identity. They were formatted to be delivered across a variety of platforms and audiences (eg, online, poster and postcards), allowing for the findings of the study and recommendations to be communicated at a wide variety of events and to be accessible to all key stakeholders with an interest in improving the mesothelioma follow-up care pathway regionally and nationally.

\section{DISCUSSION}

We have reported on the third phase of a study that aimed to explore mesothelioma patients' experiences of follow-up care in three NHS Trusts in the South of England, ${ }^{23}$ across different organisational contexts, taking into account individual patient needs, different stages of disease and treatment pathways, variations in service structures and provision, and different organisational systems and processes. ${ }^{23}$ The aim of the third consultation 


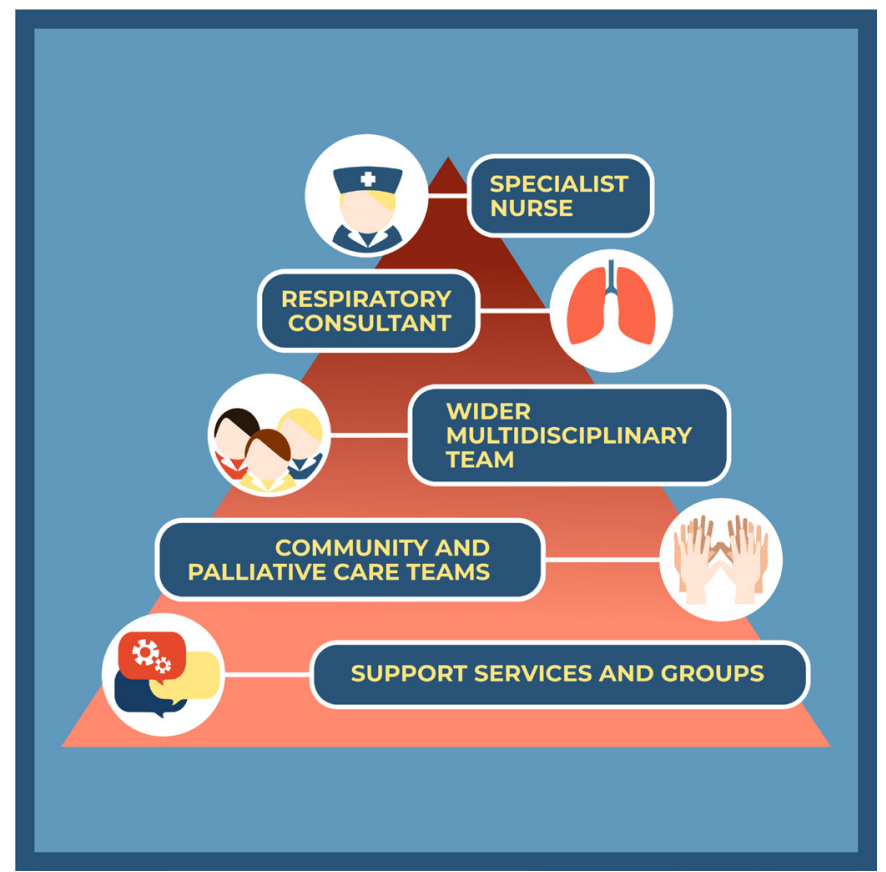

Figure 2 Pyramid of care infographic.

phase was to produce recommendations for a revised, patient-focused, mesothelioma follow-up care pathway. Three consultation group discussions were carried out with key stakeholders, specialist nurses, patients with MPM and carers, and local clinical commissioning group members, to discuss and prioritise the key themes identified in earlier phases of the study (people, processes, places, purpose and perception of care), identifying needs, barriers and solutions. Six key recommendations for developing and sustaining a streamlined, consistent, patient-centred mesothelioma follow-up care pathway were developed and a pyramid of care was conceptualised to illustrate the building blocks for best practice for patients with MPM undergoing follow-up care.

Recent research suggests that unmet needs in MPM are common, particularly around clear communication and information about the condition, follow-up care pathways, all treatment options and end-of-life and palliative care options. ${ }^{27} 28$ Moreover, variability in service provision and practice across the UK in MPM treatment and care has been observed. ${ }^{23} 2930$ The recommendations developed in this study highlight the importance of access to and communication of timely, relevant and appropriate information in line with patient needs, health literacy and stage of care. This provides support to existing guidelines and previous research, which emphasise the importance of information about treatment, disease management and accessing clinical trials opportunities, to guide decisionmaking. ${ }^{2} 1517$

Central to the development of the recommendations and the pyramid of care was the crucial role played by mesothelioma specialist nurses at all stages of follow-up care. A recent survey of healthcare professionals who treat MPM in Australia highlighted the importance of the specialist nurse roles in providing holistic and coordinated care, and information and support to patients, carers and families. ${ }^{31}$ In the current study, mesothelioma specialist nurses, properly supported by the wider multidisciplinary team, were identified as linchpins of a respiratory led mesothelioma service, providing continuity of care across the patient care pathway and promoting joined up care between primary, secondary, palliative and supportive care structures. The critically important role of the mesothelioma specialist nurse within the care pathway raises issues related to need for sufficient numbers across different geographical areas, ${ }^{17}$ and the recommendations arising from the study could be used to leverage additional funding to support these roles.

Enhancing patient-focused information resources, providing ongoing training to multidisciplinary team members and investing in additional mesothelioma specialist nurses would ensure that have patients the opportunity to access the most appropriate type, level and range of information, improve overall patient experience and help to reduce inconsistences in care and treatment provision. Where this is not feasible due to funding and resource limitations, ${ }^{17}$ it is important that the lung cancer clinical nurse specialists within the secondary care team are given a key role within the pyramid of key, allowing them to link closely with regional mesothelioma specialist nurses to optimise care.

\section{Strengths and limitations}

This study involved patients and other key stakeholders throughout the research process, and their input helped to generate and shape key recommendations and proposals for follow-up care. Adopting a co-production approach to stakeholder engagement and using the findings of the wider research study allowed for collaborative and inclusive recommendations to be developed that were also underpinned by a rigorous evidence base. In addition, a multidisciplinary approach to designing clear and well-targeted infographics (figures 1 and 2) to accompany the more detailed recommendations was a crucial step in the dissemination strategy. A lack of evaluation data has been highlighted as an ongoing problem with co-produced priority-setting approaches. ${ }^{21}$ While the current study is limited in terms of its formal evaluation of the co-production, priority setting and consensus development processes adopted, its dissemination strategy has been developed to ensure that the recommendations for policy and practice developed are promoted widely.

\section{CONCLUSION}

The recommendations developed as part of the current study are, to the best our knowledge, the first that specifically examine best practice for the follow-up care pathway for patients with MPM. The importance of co-production and public engagement in priority setting in healthcare research and to develop and optimise patientcentred care is well recognised, ${ }^{19-22}$ and combining the 
recommendations produced together with a targeted dissemination strategy and well-designed infographics will maximise their opportunity for impact regionally and nationally. Improving and streamlining the mesothelioma follow-up care pathway by focusing on continuity and joined up care, and elevating specialist mesothelioma roles and teams within a respiratory led service, have the potential to improve both the consistency and quality of care received by patients with MPM across the UK. In the long term, future research should aim to evaluate the extent to which the recommendations set out in the current study are implemented and the degree to which this improves patient experiences of follow-up care. However, in the shorter term other parts of the mesothelioma care pathway should similarly be examined to understand how mesothelioma care is developed and delivered from the point of first referral, and how clinical decision-making processes within multidisciplinary teams are developed.

\section{Twitter Catherine Henshall @cathy_henshall}

Acknowledgements We would like to thank the study participants, including patients, carers and healthcare professionals, who gave up their time to participate in this study. CH is a National Institute for Health Research (NIHR) senior nurse and midwife research leader, and also acknowledges the support of the NIHR Oxford Cognitive Health Clinical Research Facility. The views expressed in this article are those of the author(s) and not necessarily those of the NIHR, or the Department of Health and Social Care.

Contributors All authors made substantial contributions to the conception or design of the work; or the acquisition, analysis, or interpretation of data for the work and were involved in drafting the work or revising it critically for important intellectual content; gave final approval of the version to be published; have agreed to be accountable for all aspects of the work in ensuring that questions related to the accuracy or integrity of any part of the work are appropriately investigated and resolved. ZD attests that all listed authors meet authorship criteria and that no others meeting the criteria have been omitted, led on the data collection and analysis, was also responsible for collating the first draft of the paper and, as guarantor, is responsible for the overall content. $\mathrm{CH}$ was responsible for the original study design and conception, was involved in the data analysis process and the overall coordination and management of the study, and contributed to the first draft of the paper. We would like to thank members of the wider research team and the study Steering Group for their contribution to the study design and development, and $\mathrm{Dr}$ Cirenia Arias Baldrich for contributing to the development of the study infographics. We would also like to acknowledge the important contributions of the patient and carers who contributed to the study, either through their participation or through their engagement in the study design and development process.

Funding The study was funded by Mesothelioma UK. The funder was not involved in the study design or study process but were involved in reviewing and agreeing both the recommendations arising from the study and the infographics, and in the decision to submit the article for publication. The researchers confirm independence from the funders and all the authors confirm that they had full access to all of the data in the study and take responsibility for the integrity of the data and the accuracy of the data analysis.

\section{Competing interests None declared.}

Patient and public involvement Patients and/or the public were involved in the design, or conduct, or reporting, or dissemination plans of this research. Refer to the Methods section for further details.

\section{Patient consent for publication Not applicable.}

Ethics approval The study was reviewed and approved by the participating university's research ethics committees (L18170 and FREC 2018/10), the NHS Health Research Authority (19/WM/0022) and the participating trusts' local research and development departments. The Standards for QUality Improvement Reporting Excellence (SQUIRE) checklist was adhered to.

Provenance and peer review Not commissioned; externally peer reviewed.
Data availability statement Data are available upon reasonable request. Data are available on reasonable request from the corresponding author (ZD) (zdavey@ brookes.ac.uk) and is comprised of deidentified interview data from participants.

Open access This is an open access article distributed in accordance with the Creative Commons Attribution Non Commercial (CC BY-NC 4.0) license, which permits others to distribute, remix, adapt, build upon this work non-commercially, and license their derivative works on different terms, provided the original work is properly cited, appropriate credit is given, any changes made indicated, and the use is non-commercial. See: http://creativecommons.org/licenses/by-nc/4.0/.

ORCID iDs

Zoe Davey http://orcid.org/0000-0002-4583-3714

Catherine Henshall http://orcid.org/0000-0001-5659-3296

\section{REFERENCES}

1 Bibby AC, Tsim S, Kanellakis N, et al. Malignant pleural mesothelioma: an update on investigation, diagnosis and treatment. Eur Respir Rev 2016;25:472-86.

2 Woolhouse I, Bishop L, Darlison L, et al. British thoracic Society guideline for the investigation and management of malignant pleural mesothelioma. Thorax 2018;73:11-30.

3 Cancer Research UK. Statistics by cancer type, 2021. Available: https://www.cancerresearchuk.org/health-professional/cancerstatistics/statistics-by-cancer-type/lung-cancer - heading-Zero

4 Cancer Research UK. Mesothelioma incidence trends over time, 2021. Available: https://www.cancerresearchuk.org/ health-professional/cancer-statistics/statistics-by-cancer-type/ mesothelioma/incidence\#heading-Two

5 Scherpereel A, Astoul P, Baas P, et al. Guidelines of the European respiratory Society and the European Society of thoracic surgeons for the management of malignant pleural mesothelioma. Eur Respir $J$ 2010;35:479-95.

6 Chapman E, Hughes D, Landy A, et al. Challenging the representations of cancer pain: experiences of a multidisciplinary pain management group in a palliative care unit. Palliat Support Care 2005;3:43-9.

7 Cordes ME, Brueggen C. Diffuse malignant pleural mesothelioma: Part II. symptom management. Clin J Oncol Nurs 2003;7:545-52.

8 Clayson H, Seymour J, Noble B. Mesothelioma from the patient's perspective. Hematol Oncol Clin North Am 2005;19:1175-90.

9 Pistolesi M, Rusthoven J. Malignant pleural mesothelioma: update, current management, and newer therapeutic strategies. Chest 2004;126:1318-29.

10 Ball H, Moore S, Leary A. A systematic literature review comparing the psychological care needs of patients with mesothelioma and advanced lung cancer. Eur J Oncol Nurs 2016;25:62-7.

11 British Lung Foundation. Survey of mesothelioma patients and their carers. London: British Lung Foundation, 2009.

12 Clayson H. Suffering in mesothelioma: concepts and contexts. Prog Palliat Care 2003:11:251-5.

13 Hughes N, Arber A. The lived experience of patients with pleural mesothelioma. Int J Palliat Nurs 2008;14:66-71.

14 Lebovits AH, Chahinian AP, Gorzynski JG, et al. Psychological aspects of asbestos-related mesothelioma and knowledge of high risk for cancer. Cancer Detect Prev 1981;4:181-4.

15 Walker SL, Crist JD, Shea K, et al. The lived experience of persons with malignant pleural mesothelioma in the United States. Cancer Nurs 2021;44:E90-8.

16 Darlison L. Improving mesothelioma services. Lung Cancer in Practice 2008;5:10-11.

17 Darlison L, Mckinley D, Moore S. Findings from the National mesothelioma experience survey. Cancer Nursing Practice 2014;13:32-8.

18 Woolhouse I, Bishop L, Darlison L, et al. BTS guideline for the investigation and management of malignant pleural mesothelioma. BMJ Open Respir Res 2018;5:e000266.

19 NHS Leadership Academy. Co-production and community development: a primer, 2018. Available: https://www. leadershipacademy.nhs.uk/wp-content/uploads/2019/01/ Community-development-and-coproduction-a-primer_FINAL.pdf

20 Turakhia P, Combs B. Using principles of Co-Production to improve patient care and enhance value. AMA J Ethics 2017;19:1125-31.

21 Manafò E, Petermann L, Vandall-Walker V, et al. Patient and public engagement in priority setting: a systematic rapid review of the literature. PLoS One 2018;13:e0193579.

22 NHS Improvement. Valued care in mental health: improving for excellence, 2018. Available: https://improvement.nhs.uk/documents/ 3453/NHS_Mental_Health_Improvement_web.pdf 
23 Henshall C, Davey Z, Walthall $\mathrm{H}$, et al. Recommendations for improving follow-up care for patients with mesothelioma: a qualitative study comprising documentary analysis, interviews and consultation meetings. BMJ Open 2021;11:e040679.

24 Gale NK, Heath G, Cameron E, et al. Using the framework method for the analysis of qualitative data in multi-disciplinary health research. BMC Med Res Methodol 2013;13:117.

25 Ritchie J, Spencer L. Qualitative data analysis for applied policy research. In: Bryman A, Burgess R, eds. Analysing qualitative data. London: Routledge, 1993.

26 Murray IR, Murray AD, Wordie SJ, et al. Maximising the impact of your work using infographics. Bone Joint Res 2017;6:619-20.

27 Hoon SN, Lawrie I, Qi C, et al. Symptom burden and unmet needs in malignant pleural mesothelioma: exploratory analyses from the RESPECT-Meso study. J Palliat Care 2021;36:113-20.
28 Warby A, Dhillon HM, Kao S, et al. A survey of patient and caregiver experience with malignant pleural mesothelioma. Support Care Cancer 2019a;27:4675-86.

29 Harden SV, Darlison L, Beckett P, et al. Standards of care in mesothelioma treatment. Br J Cancer 2020;123:1588-9.

30 Bibby AC, Williams K, Smith S, et al. What is the role of a specialist regional mesothelioma multidisciplinary team meeting? A service evaluation of one tertiary referral centre in the UK. BMJ Open 2016;6:e012092.

31 Warby A, Dhillon HM, Kao S, et al. Managing malignant pleural mesothelioma: experience and perceptions of health care professionals caring for people with mesothelioma. Support Care Cancer 2019b;27:3509-19. 